Check for updates

Cite this: RSC Adv., 2017, 7, 23820

Received 22nd February 2017

Accepted 24th April 2017

DOI: 10.1039/c7ra02215h

rsc.li/rsc-advances

\section{Conformable and ionic textiles using sheath-core carbon nanotube microyarns for highly sensitive and reliable pressure sensors $\uparrow$}

\begin{abstract}
So Young Kim, ${ }^{\text {a Eunsong Jee, }}$, Joo Sung Kim ${ }^{\text {ab }}$ and Do Hwan Kim (D) *ab
In order to exploit large-area and conformable electronic textile (e-textile) pressure sensors that are consistently sensitive to a wide range of pressures, both superior electrical and mechanical properties such as high conductivity and high flexibility are strongly required in individual yarns, but remain unexplored in tactile sensor research. In this report, we demonstrate the first highly sensitive, conformable and reliable ionic textile (i-textile) pressure sensors using sheath-core carbon nanotube (CNT) microyarns encapsulated with ionic thermoplastic polyurethane (i-TPU) dielectrics. The piezocapacitive i-textile pressure sensors that are formed by weaving two distinct sets of microyarns (CNT microyarns and i-TPU/CNT microyarns), were capable of simultaneously detecting subtle pressure and human touch with a high sensitivity of $1 \mathrm{nF} \mathrm{kPa}{ }^{-1}$ and operational reliability over a wide range of pressure, even under low-voltage. We believe that the conformable i-textile pressure sensors suggested by us will be a promising platform to achieve human-adaptive wearable textronics capable of perceiving health monitoring as well as real-time human motion.
\end{abstract}

\section{Introduction}

Recently, rational design of electronic textiles (e-textiles) that can be conformably contacted onto curved or wrinkled surfaces, has been a big challenge in wearable applications such as energy harvesting devices, ${ }^{1-4}$ sensors,${ }^{5,6}$ batteries ${ }^{7,8}$ and transistors. ${ }^{9}$ Further, beyond any conventional bulky or planar structures, utilization of e-textiles would make an unprecedented impact on the field of wearable optoelectronics because etextiles are, in general, composed of smart yarns that can be effectively adapted to complex or undesired deformations, while maintaining high-performance and high-reliability. ${ }^{10,11}$ In particular, e-textiles-based pressure sensors have been widely explored for a variety of applications to include human-adaptive caring for the elderly, ${ }^{12}$ diagnostics,${ }^{13}$ monitoring patients ${ }^{14}$ and human motion detection. ${ }^{15}$ However, to exploit large-area, highly sensitive and wearable tactile system using e-textiles, superior electrical or mechanical properties such as high conductivity and high flexibility are strongly required in individual conducting yarns. ${ }^{16}$ This is due to severe textile forming processes including knitting, weaving and braiding. ${ }^{17}$ Progress

\footnotetext{
${ }^{a}$ Department of Organic Materials and Fiber Engineering, Soongsil University, 369 Sangdo-Ro, Dongjak-Gu, Seoul 06978, Korea. E-mail: dohwan@ssu.ac.kr

${ }^{b}$ Department of Information Communication, Materials, and Chemistry Convergence Technology (BK-21 plus), Soongsil University, 369 Sangdo-Ro, Dongjak-Gu, Seoul 06978, Korea
}

$\dagger$ Electronic supplementary information (ESI) available. See DOI: $10.1039 / \mathrm{c} 7 \mathrm{ra02215h}$ has been extensively made regarding to this issue by developing two representative strategies to manufacture flexible or even deformable conducting yarns for e-textiles-based tactile platform: (i) conductive layer can be formed onto conventional polymer yarns such as polyurethane, cotton and nylon via dipcoating, electro/electroless-plating and chemical vapour deposition (CVD) ${ }^{18-29}$ (ii) Highly flexible and even elastic conducting yarns such as carbon nanotubes (CNTs) and poly(3,4ethylenedioxy-thiophene):polystyrene sulfonate (PEDOT:PSS) that were blended with elastomer can be fabricated by direct spinning process..$^{30-32}$ These approaches have reflected good conductivity and appropriate mechanical properties as yarn electrodes.

Despite these accomplishments, however, the aforementioned methodologies show several drawbacks, such as poor reliability and uniformity due to stepwise complicated processes, which may not serve the ultimate solution leading to reliable and wearable conducting yarns for achieving highly sensitive and conformable pressure sensors. As a more impactful system, CNT yarns have attracted considerable interests due to their advantages such as low density, cost effectiveness, excellent electrical conductivity, flexibility and scalability. ${ }^{33-36}$ This implies that CNT yarns are in high demand for scalable and stretchable conductors that are expected to be stretched and conformable in a 3-dimensional structure. ${ }^{37}$

Moreover, a great care for selecting device types of e-textilesbased pressure sensors would be required to be optimal for targeting high-performance. Typically, a piezocapacitive pressure sensor shows lots of favorable aspects such as high 
sensitivity, excellent stability, fast response time and low power consumption compared to its piezoresistive counterpart. ${ }^{\mathbf{3 8 - 4 0}}$ The e-textile-based piezocapacitive pressure sensor can be conventionally fabricated by utilizing two compliant conducting yarns as electrodes, while separated by deformable dielectric spacers such as foams, spacers and soft polymers. ${ }^{41,42}$ However, conventional dielectrics suffer from exhibiting highcapacitance under electrical bias. Recently, Nie et al. demonstrated iontronic film-based pressure sensors with ultrahigh mechanical-to-capacitive sensitivity of $3.1 \mathrm{nF} \mathrm{kPa}^{-1}$ by allowing highly capacitive interfaces at the ionic-electronic contact. ${ }^{43}$ The interfacial capacitance lies on the formation of an electrical double layer (EDL), in which electrons on the electrode and the counter ions from the iontronic film accumulate and attract to each other at a nanoscopic distance. As a result, this leads to an ultrahigh unit-area capacitance. Despite successful demonstration of highly sensitive ionic pressure sensors under mechanical stimuli, the resulting implementations are still vulnerable to undesired deformation, thereby preventing conformable contact of the devices onto curved or wrinkled surfaces.

Here, we describe the first highly conformable and reliable ionic textiles (i-textiles) on the basis of sheath-core carbon nanotube (CNT) microyarns to implement highly sensitive and reliable piezocapacitive pressure sensor arrays. An ionic thermoplastic polyurethane (i-TPU) dielectric, which is capable of effectively sensing pressure in woven textiles, was formed onto conducting CNT microyarns as an ionic sheath material so as to allow low-voltage operation and high flexibility as well as high sensitivity. This is originated from an increase in contact area and an effective ion migration at the interface of CNT microyarn and ionic CNT microyarns with sheath-core microstructure (iTPU/CNT) under pressure. ${ }^{44}$ Potentially, conformable i-textilesbased pressure sensors suggested by us exhibits high sensitivity of $1 \mathrm{nF} \mathrm{kPa}{ }^{-1}$ and operational stability over a wide range of pressure, enabling conditions conducive to real-time human motion detection.

\section{Experimental}

\subsection{Synthesis of CNT microyarns by dry CVD}

A schematic of the overall fabrication process is depicted in Fig. S2. $\dagger$ CNT microyarns are synthesized by dry chemical vapour deposition (CVD) method followed by wet spinning in water bath. Ferrocene and thiophene were purchased from Sigma Aldrich, which were used as received. The synthesis was conducted by injecting a mixture solution with a hydrogen gas flow into a heated furnace. ${ }^{36}$ CNT web was descended into a water bath at one end of the furnace, and then wound at a rate of $5 \mathrm{~m} \mathrm{~min}{ }^{-1}$. Standard procedure for obtaining conductive CNT microyarn was taken to be acetone of $98.0 \mathrm{wt} \%$, ferrocene of $1.0 \mathrm{wt} \%$, and thiophene of $1.0 \mathrm{wt} \%$ injected at a $10 \mathrm{ml} \mathrm{h}^{-1}$ rate into the carrier hydrogen gas $(1000 \mathrm{sccm})$ at $1200{ }^{\circ} \mathrm{C}$. The composition of precursor solution and the other parameters were optimized until reaching a range in which CNT microyarn could be spun continuously.

\subsection{Preparation of i-TPU solution and ionic CNT microyarn} with sheath-core microstructure

Thermoplastic polyurethane (TPU) chips (KA-480) were purchased from KOLON INDUSTRIES, INC. 1-Ethyl-3-methylimidazolium:bis(trifluoromethylsulfoyl)imide [EMIM $]^{+}[\mathrm{TFSI}]^{-}$and DMF ( $N, N$-dimethylformamide) solvent were supplied by SigmaAldrich. First, TPU chips were dissolved into DMF (mass ratio: 1.5 : 5), and the solution was stirred at $60{ }^{\circ} \mathrm{C}$ for 3 hours. Then, 20 $\mathrm{wt} \%[\mathrm{EMIM}]^{+}[\mathrm{TFSI}]^{-}$on the weight of TPU was added into the prepared solution for further stirring $\left(60{ }^{\circ} \mathrm{C}\right.$ and 2 hours $) .{ }^{44}$ Ionic CNT microyarns with sheath-core microstructure (i-TPU/CNT) were fabricated using a well-controlled die-coating method. A well-mixed ionic liquid and a TPU solution were poured into silicon bath. Then, CNT microyarns were coated by i-TPU at a rate of $10 \mu \mathrm{m} \mathrm{s}^{-1}$ and cured in an oven at $120{ }^{\circ} \mathrm{C}$. During curing process, i-TPU/CNT microyarns were placed under a vacuum for 24 hours to fully remove a residual solvent. i-TPU/CNT microyarns were then baked at $125^{\circ} \mathrm{C}$ for $35 \mathrm{~min}$ to improve their mechanical properties.

\subsection{Characterization of CNT and ionic CNT microyarns}

The surface morphological features of CNT microyarns were characterized by field-emission scanning electron microscopy (FESEM, JSM-6700F, JEOL). The mechanical property of CNT and i-TPU/CNT microyarns was measured using a universal tensile machine (Instron, model 4464). The sample gauge length and rate of extension were $10 \mathrm{~mm}$ and $3 \mathrm{~mm} \mathrm{~min}{ }^{-1}$, respectively. Also, the electrical conductivity of CNT and i-TPU/ CNT microyarns was measured using mX HiTESTER model from HIOKI using the 4-point probes method.

\subsection{Characterization of woven i-textile pressure sensors}

To observe the responses of piezocapacitive i-textile pressure sensors that are formed by weaving two distinct sets of microyarns (CNT microyarns and i-TPU/CNT microyarns) against multiple stimuli, resistance and capacitance were measured using a precision LCR meter (E4980a, Agilent Technologies). Pressure sensing capability of i-textile pressure sensors was characterized by using a custom-built sensor probe station with a programmable $x y$ - and $z$-axis stage $(0.1 \mu \mathrm{m}$ resolution), a force gauge (Mark-10) and all interfaced through a computer (Fig S6, ESI $\dagger$ ). The force gauge measures the applied load, and corresponding pressure was calculated by dividing the probe with the area. Also, the sensitivity of the device was identified with the slope of capacitance versus the pressure variation $\left(\left(\Delta C / C_{0}\right) / \Delta P\right)$. The measuring equipment was connected to the customized lab view-based program, and this system simultaneously recorded the in situ capacitance change and the loaded pressure.

\section{Results and discussion}

Fig. 1a presents a photograph and a schematic view of wearable i-textiles formed by weaving two distinct sets of microyarns (CNT microyarns and ionic CNT microyarns (i-TPU/CNT)) together, and molecular compounds that constitute i-TPU sheath introduced in i-TPU/CNT microyarns. The i-textiles-based pressure 
(a)

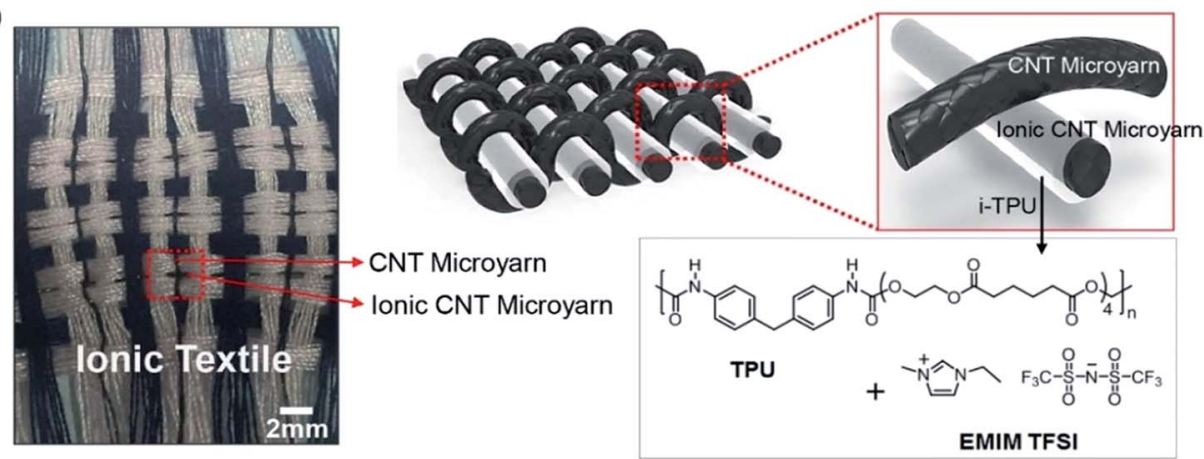

(b)

(c)
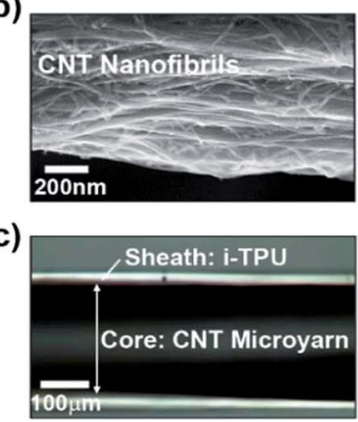

(d)

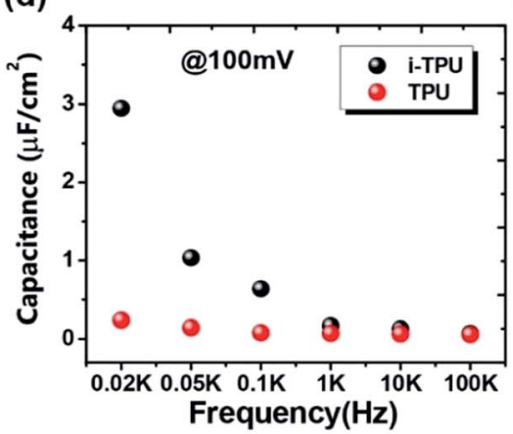

(e)

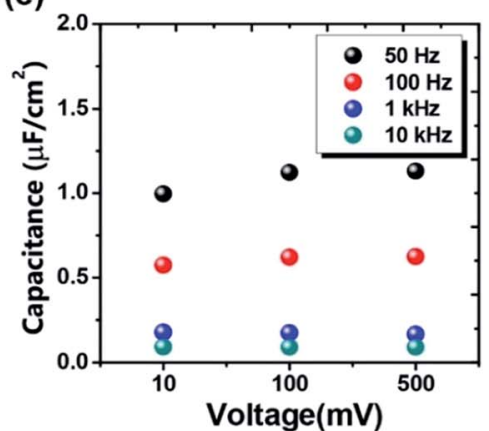

Fig. 1 (a) A photograph and a schematic view of wearable i-textiles formed by weaving two distinct sets of microyarns (CNT microyarns and ionic CNT microyarns (i-TPU/CNT)) together, and molecular components of i-TPU. (b) A FE-SEM image of CNT microyarn surface. (c) A optical microscopy image of sheath-core microstructured ionic CNT microyarns. (d) $C-f$ characteristics of the (ionic and non-ionic) textile capacitors upon varying the types of sheath materials (i-TPU and TPU). (e) $\mathrm{C}-\mathrm{V}$ characteristics of the i-textile capacitor in terms of the frequencies (50 Hz, $100 \mathrm{~Hz}, 1 \mathrm{kHz}$ and $10 \mathrm{kHz}$ ).

sensor clearly exhibits mechanical robustness under various mechanical deformations such as crumpling and stretching conditions (Fig. S1, ESI $\dagger$ ). The piezocapacitive pressure sensor is operated at the cross point of two distinct sets of CNT microyarns as shown in Fig. 1a (upper right). Typically, to achieve welldefined and low-power i-textiles, highly conductive and elastic microyarns are required. ${ }^{16}$ As mentioned in our previous work, ${ }^{36}$ we have utilized CNT microyarns that are synthesized by dry chemical vapour deposition (CVD) method, direct spinning from a carbon source such as acetone with ferrocene as a catalyst, and thiophene as a promoter (Fig. S2, ESI†). As shown in Fig. 1b, CNT microyarn surface displays a hierarchically structured fibrous network, originated from sequential self-organization of the individual CNTs ((i) individual multi-walled carbon nanotube (MWNT) (ii) CNT bundle (iii) bundle network (iv) CNT microyarn) during CVD synthesis and winding process (Fig. S2, ESI†). ${ }^{36}$ Fig. 1c shows the optical microscopy image of i-TPU/CNT microyarns, representing sheath-core microstructure. The thickness of i-TPU dielectric (used as a sheath layer) was about $\sim 30 \mu \mathrm{m}$, showing clear interface with CNT microyarn (used as a core part). In this work, i-TPU dielectric was formed by the non-covalent association of 1-ethyl-3-methylimidazolium bis(trifluoromethyl-sulfonyl)imide ([EMIM $]^{+}[\mathrm{TFSI}]^{-}$cationanion pairs) loaded in TPU matrix, representing high elasticity and high transparency even under stretchy (Fig. S3, ESI † Furthermore, both CNT and i-TPU/CNT microyarns intrinsically exhibit high conductivity of $10^{6} \mathrm{~S} \mathrm{~m}^{-1}$ and tensile strain of even
15\% (Fig. S4, ESI†), which might be feasible to the implementation of wearable and low-power i-textiles via weaving processes. Note that it is envisaged that elastic i-TPU sheath layer can improve mechanical properties of i-TPU/CNT microyarns via sheath-core microstructure, which would lead to enhanced reliability of i-textiles.

To determine and optimize the electrical properties of $\mathrm{i}$ textile capacitors woven by two distinct sets of CNT microyarns (CNT microyarns and i-TPU/CNT microyarns) under an electric field, the devices upon varying the types of sheath materials (i-TPU and TPU) are evaluated. Fig. 1d shows $C-f$ characteristics of (ionic and non-ionic) textile capacitors, in which a high capacitance of up to $3 \mu \mathrm{F} \mathrm{cm}{ }^{-2}$ for i-textile devices was observed at a low frequency of $20 \mathrm{~Hz}$. This is attributed to the EDL formation at the interface of i-TPU and CNT microyarns. It is found that this behaviour is also observed in $C-f$ characteristics of i-TPU film that is embedded into Au-coated wafer (Fig. S5, ESI $\dagger$ ). In addition, to elucidate voltage dependence of i-textile capacitors, we investigated $C-V$ characteristics in terms of the frequencies $(50 \mathrm{~Hz}, 100 \mathrm{~Hz}, 1 \mathrm{kHz}$ and $10 \mathrm{kHz})$. In Fig. 1e, we could observe that the capacitance slightly increases upon relatively high voltage in low frequency region.

The pressure sensing capability of piezocapacitive textile pressure sensors, which are formed by weaving two distinct sets of microyarns (CNT microyarns and i-TPU/CNT or TPU/CNT microyarns), was examined by applying a normal force to the unit sensing cell using a custom-built sensor probe station 
(Fig. S6, ESI $\dagger$ ). Fig. 2a shows the capacitance change $\left(\Delta C / C_{0}\right)$ depending on the applied normal pressure upon varying the types of sheath materials (i-TPU and TPU). As shown in Fig. 2a, the sensitivity of the pressure sensor that is fabricated with CNT and TPU/CNT microyarns shows a typical saturation behaviour (0.01 $\mathrm{pF} \mathrm{kPa}^{-1}$ below $10 \mathrm{kPa}$ and $0.001 \mathrm{pF} \mathrm{kPa}{ }^{-1}$ over $10 \mathrm{kPa}$ ) while the pressure sensor woven with CNT and i-TPU/CNT microyarns exhibits a remarkable sensitivity $\left(0.68 \mathrm{nF} \mathrm{kPa}{ }^{-1}\right.$

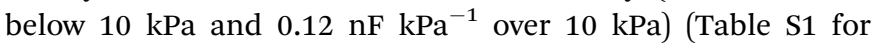
details, ESI $\dagger$ ). In other words, the pressure-to-capacitance sensitivity could be improved by a factor of 68 from $0.01 \mathrm{pF}$ $\mathrm{kPa}^{-1}$ to $0.68 \mathrm{nF} \mathrm{kPa}^{-1}$ below $10 \mathrm{kPa}$, and by a factor of 120 from $0.001 \mathrm{pF} \mathrm{kPa}^{-1}$ to $0.12 \mathrm{nF} \mathrm{kPa}{ }^{-1}$ over $10 \mathrm{kPa}$. In addition, as seen in Fig. 2b, the sensitivity could be optimized to $1.0 \mathrm{nF} \mathrm{kPa}{ }^{-1}$ below $10 \mathrm{kPa}$ by precisely tuning the measurement frequency (Table S2 for details, ESI $\dagger$ ). In particular, in order to explain this unprecedented high sensitivity of i-textile pressure sensors that are formed by weaving two distinct sets of microyarns (CNT microyarns and i-TPU/CNT microyarns), we can describe a schematic diagram showing the change of contact area between CNT microyarn and i-TPU/CNT micro yarn before and after applying pressure in Fig. 2c, in which the contact area increases upon applying pressure. Also, Fig. 2d shows operating mechanisms of textile pressure sensors, which can be depicted in terms of types of the sheath materials (TPU and i-TPU). Typically, non-ionic textile pressure sensors can sense an increase of capacitance under pressure as the thickness of TPU dielectric is decreased upon an increase of pressure (Fig. 2d, left). On the other hand, in case of ionic textile pressure sensors, the capacitance can be improved by increasing the contact area between CNT microyarn and i-TPU/CNT microyarn under pressure unlike non-ionic one. This is due to EDL working mechanism sensitive to contact area of electrode and dielectric. ${ }^{\mathbf{4 3 , 4 4}}$ Furthermore, as mentioned in our previous work, ${ }^{\mathbf{4 4}}$ ion pairs can be squeezed out from the free volume of i-TPU matrix to a contact with CNT microyarns by visco-poroelasticity through molecular confinement in TPU chains under pressure. As a consequence, when a higher pressure is applied to iTPU sheath, a larger amount of ion pairs is squeezed out to EDL with a densely charged electrode by the interplay of viscoelastic deformation of TPU chains and poroelasticity of ionic liquids under pressure. These i-textile pressure sensors with viscoporoelasticity have been found to enable a remarkable sensitivity to adapt to a wide range of pressures, with a daily pressure of up to $150 \mathrm{kPa}$. (a)

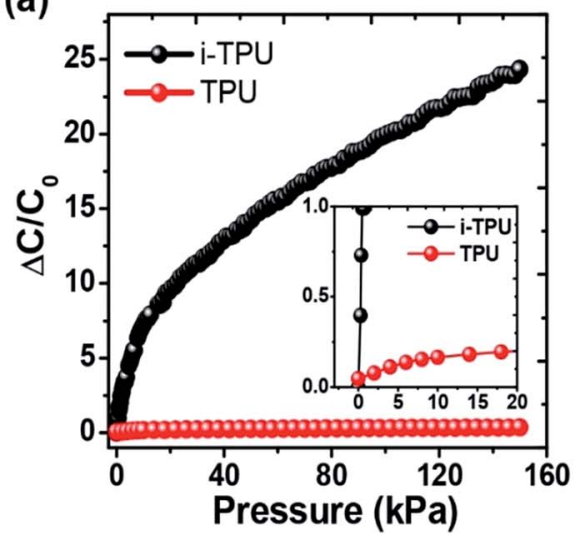

(b)

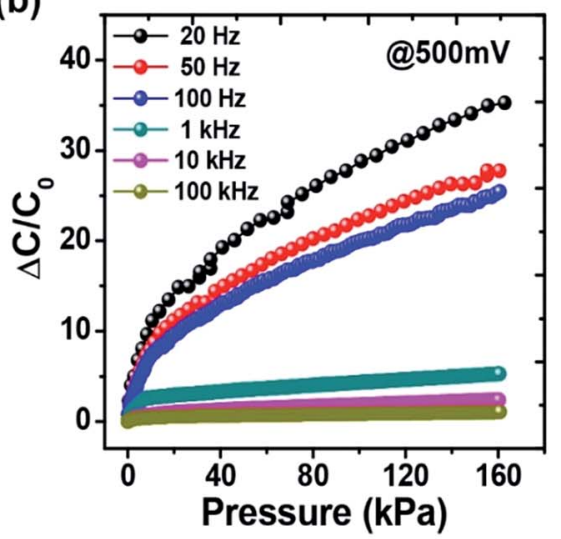

(c)

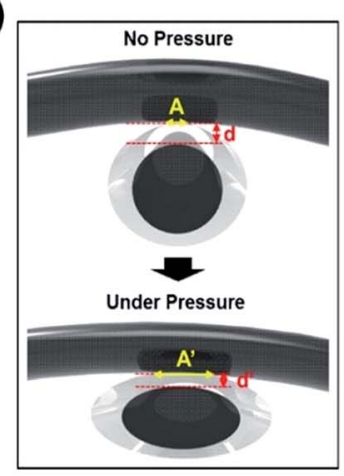

(d)

\section{TPU Sheath}

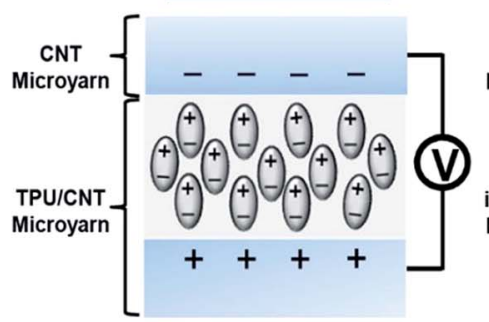

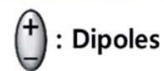

\section{i-TPU Sheath}

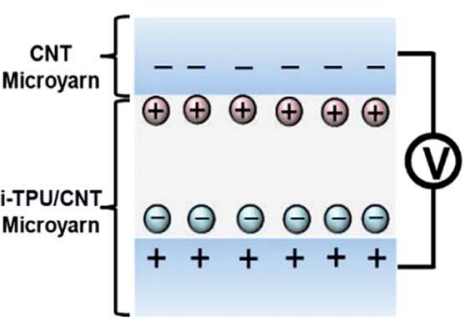

$\Theta$ : Anions

$($ ) : Cations

Fig. 2 Pressure sensing capability of piezocapacitive sensor based on i-textiles. (a) Relative capacitance change $\left(\Delta C / C_{0}\right)$ versus normal pressure for ionic and non-ionic textile pressure sensors. (b) Relative capacitance change $\left(\Delta C / C_{0}\right)$ versus normal pressure as a function of the measurement frequencies. (c) A schematic diagram showing the change of contact between CNT microyarn and sheath-core microstructured ionic CNT microyarn before and after applying pressure in the pressure sensor geometry. (d) Operating mechanisms of non-ionic (left) and ionic (right) textile pressure sensors in terms of the types of the sheath materials (TPU and i-TPU). 
The capacitive response of i-textile pressure sensors was also found to be highly reversible. Interestingly, a high stability for the change in capacitance was obtained in response to the dynamic pressure (1 kPa and $10 \mathrm{kPa}$ ) under $500 \mathrm{mV}$ (Fig. 3a and b). As shown in Fig. 3a, when a pressure is continuously applied to i-textile pressure sensors, the contact area of i-TPU sheath between two CNT microyarns increases, resulting in an increase in capacitance by EDL mechanism. Typically, in case of ionic capacitor system, it is well known that capacitance is less sensitive to thickness change compared to contact area. ${ }^{44}$ On the other hand, the resistance of CNT microyarns was almost unchanged even under pressure (Fig. S7, ESI $\dagger$ ), implying that the capacitance modulation is only originated from change of contact area (i.e. mechanical deformation) of i-TPU dielectric. We also observed the good durability of our device as a pressure sensor by measuring the capacitance of the device when repeatedly load/unload applied pressures of $1 \mathrm{kPa}$ and $10 \mathrm{kPa}$ for more than 2000 cycles in Fig. 3b, and by measuring the response of the device for 80 cycles pressure sensing under stepwise conditions ( $0.1 \mathrm{kPa}, 1 \mathrm{kPa}, 5 \mathrm{kPa}$ and $10 \mathrm{kPa})$ in Fig. 3c. For i-textile pressure sensors, Fig. 3d describes an instant response of $78 \mathrm{~ms}$ that can be detected from $1 \mathrm{kPa}$, but a somewhat longer reset time of $312 \mathrm{~ms}$ was found, which might be a result of the relaxation issue of the polymer chains in i-TPU sheath right after eliminating the pressure. Fig. 3e shows a transient response of the device upon the placement and removal of a small weight such as a paper sheet on the sensor pixel, corresponding to low pressures of $23 \mathrm{~Pa}$ and $15 \mathrm{~Pa}$, respectively.

To demonstrate the spatial distribution of a proof-of-concept conformable i-textile pressure sensor array against an input pressure signal, we incorporated individual CNT and i-TPU/CNT microyarns into cotton fabrics, fabricating $3 \times 3$ sensing arrays of 9 pixels. Fig. 4a shows images and two-dimensional (2D) $\Delta C /$ $C_{0}$ map of the sensor array with wooden doll $(1.5 \mathrm{~g})$ and plastic rob $(3 \mathrm{~g})$ placed on selective locations, respectively. The spatial distribution of input pressure signals is clearly identified, even though a slight crosstalk between the pressed and non-pressed pixels occurs. As shown in Fig. 4b, human motion could be also detected in i-textile pressure sensor array that is associated with forefinger motion before and after bending. When the forefinger was bent, the capacitance change clearly increased by effective pressure. Notably, the capacitance change reliably returned to the initial value once the forefinger was fully unfolded. This reflects excellent reliability of our i-textile pressure sensors for human motion detection. Furthermore, we intend to evaluate pressure and touch sensing capability using i-textiles woven by CNT and i-TPU/CNT microyarns. Fig. 4c shows images and $\Delta C / C_{0}$ response of woven i-textile pressure sensor with minute paper star $(30 \mathrm{mg})$ and thin polymer film (50

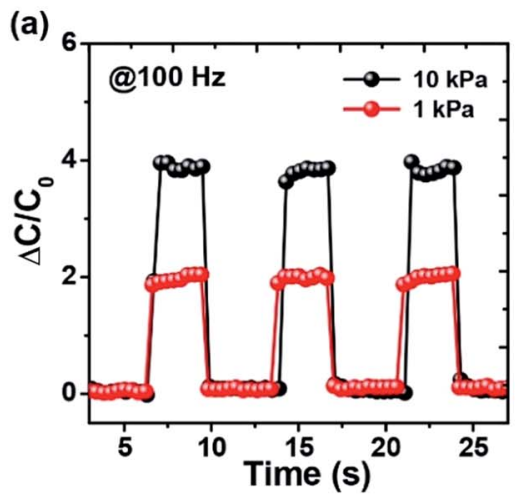

(d)

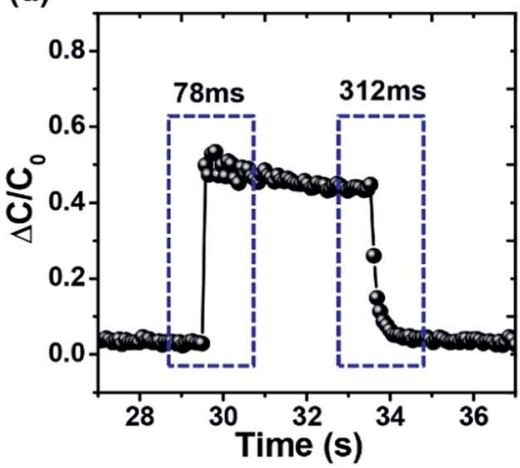

(b)

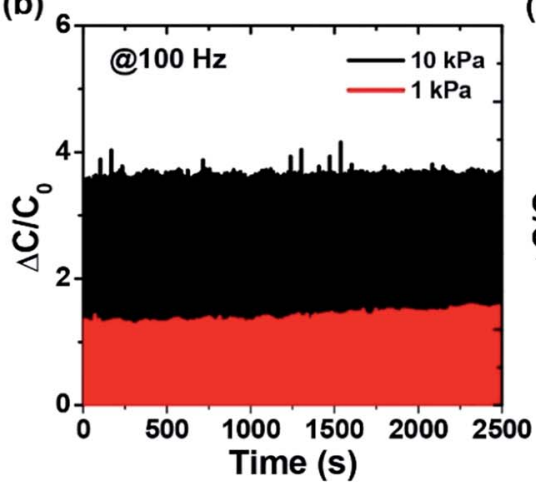

(e)

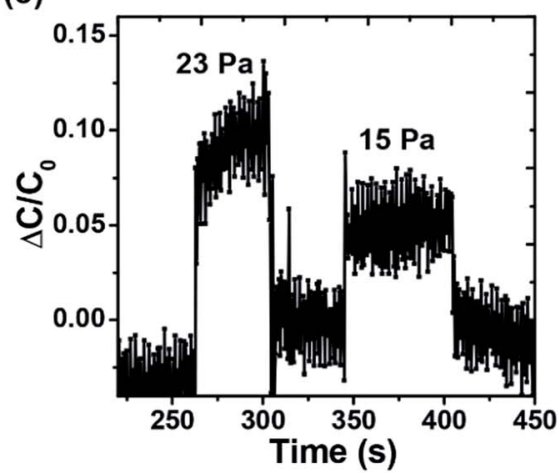

Fig. 3 Dynamic pressure sensing capability of piezocapacitive sensor based on i-textiles. (a) $\Delta C / C_{0}$ versus time over three cycles of applied pressure. (b) Durability test under a pressure of $1 \mathrm{kPa}$ and $10 \mathrm{kPa}$. (c) Response of i-textile pressure sensor for 80 cycles pressure sensing with stepwise conditions $(0.1 \mathrm{kPa}, 1 \mathrm{kPa}, 5 \mathrm{kPa}$ and $10 \mathrm{kPa})$. (d) Transient response and reset time of i-textile pressure sensor, representing a response time of $78 \mathrm{~ms}$ and a reset time of $312 \mathrm{~ms}$. (e) Transient response to the application and removal of a paper sheet on the sensor pixel, corresponding to a pressure of only $23 \mathrm{~Pa}$ and $15 \mathrm{~Pa}$, respectively. 
(a)

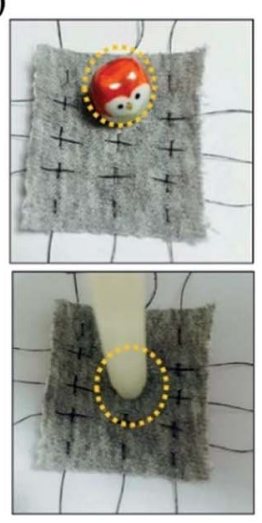

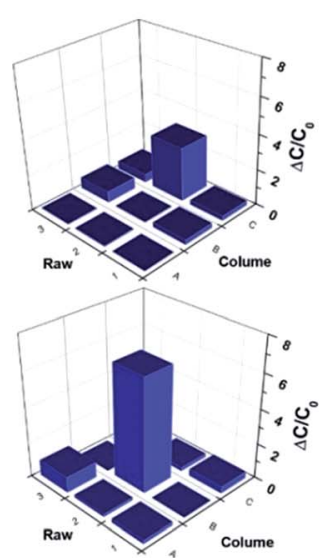

(b)

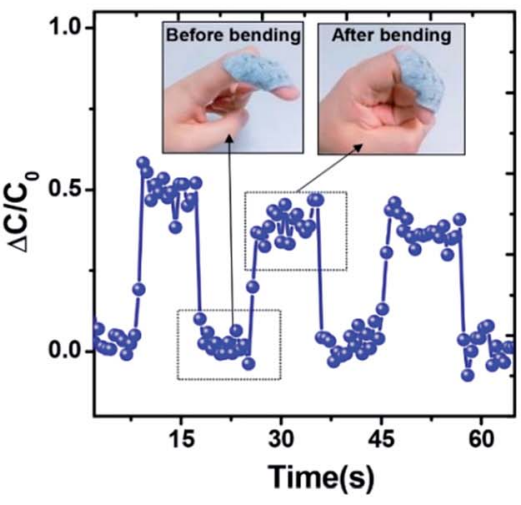

(c)

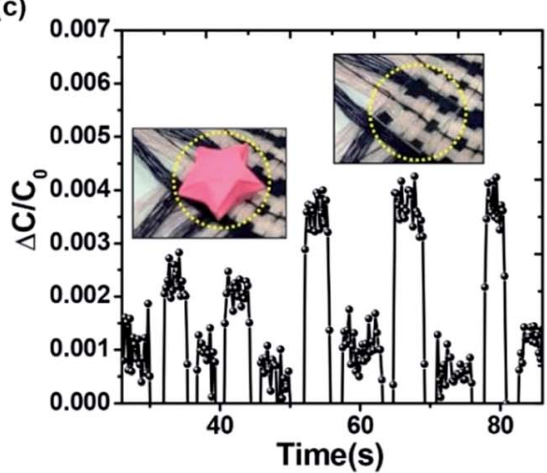

(d)

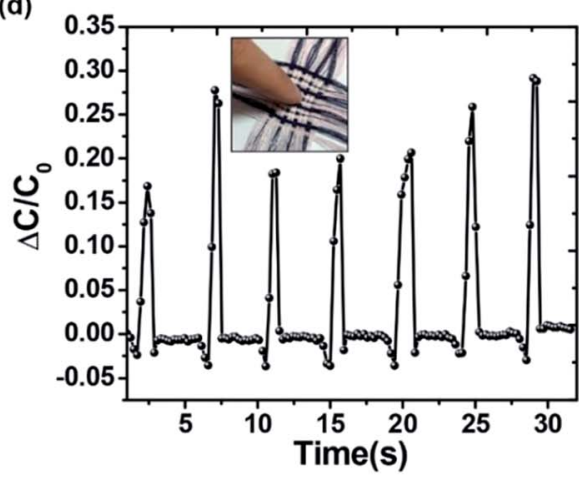

Fig. 4 (a) Spatial distribution of an input pressure signal onto integrated $3 \times 3$ i-textile pressure sensor array. Images and two-dimensional intensity $\left(\Delta C / C_{0}\right)$ map of the i-textile pressure sensors with minute wooden doll $(1.5 \mathrm{~g})$ and plastic rob $(3 \mathrm{~g})$ placed on selective locations for pressure sensing. (b) Detection of human motion onto i-textile pressure sensor array. Relative capacitance change associated with forefinger motion before and after bending. (c) Woven i-textiles capable of perceiving a minute input pressure signal by paper star (30 mg) and thin polymer film (50 mg) placed on selective locations. (d) Woven i-textiles capable of perceiving the change of capacitance before and after gentle touch stimuli.

$\mathrm{mg}$ ) placed on selective locations, which successfully perceives a change of weight. As shown in Fig. 4d, besides pressure sensing capabilities, woven i-textile pressure sensors were investigated to discern human touch from other gentle pressure, in which woven i-textiles can generate the change of capacitance before and after gentle touch stimuli.

\section{Conclusions}

In conclusion, we have developed the first highly sensitive, conformable and reliable i-textile pressure sensors on the basis of sheath-core CNT microyarns coupled with i-TPU dielectrics. The piezocapacitive i-textile pressure sensors, which are formed by weaving two distinct sets of microyarns (CNT microyarns and i-TPU/CNT microyarns), were capable of detecting subtle pressure and human touch with high sensitivity of $1 \mathrm{nF} \mathrm{kPa}{ }^{-1}$ and operational reliability over a wide range of pressure, even under low-voltage. This is attributed to a change in contact area and an effective ion migration at the interface of mechanically deformable CNT microyarn and i-TPU/CNT microyarn under pressure. We also confirmed good durability of our i-textiles as pressure sensors by measuring the capacitance of the device when repeatedly load/unload an applied pressure of $1 \mathrm{kPa}$ and $10 \mathrm{kPa}$ for more than 2000 cycles. In addition, based on this type of highly conformable and reliable i-textiles, forefinger motion before and after bending could be effectively detected in i-textile pressure sensor array. We believe that engineering conformable i-textiles for tactile responses will be an effective way to achieve human-adaptive wearable electronics.

\section{Acknowledgements}

This work was supported by the Soongsil University Research Fund by 2012 .

\section{References}

1 W. Zeng, X. M. Tao, S. Chen, S. Shang, H. L. W. Chan and S. H. Choy, Energy Environ. Sci., 2013, 6, 2631-2638.

2 Y. Qin, X. Wang and Z. L. Wang, Nature, 2008, 451, 809-813. 3 M. Lee, C. Y. Chen, S. Wang, S. N. Cha, Y. J. Park, J. M. Kim, L. J. Chou and Z. L. Wang, Adv. Mater., 2012, 24, 1759-1764. 4 X. Li, Z. H. Lin, G. Cheng, X. Wen, Y. Liu, S. Niu and Z. L. Wang, ACS Nano, 2014, 8, 10674-10681.

5 J. Lee, H. Kwon, J. Seo, S. Shin, J. H. Koo, C. Pang, S. Son, J. H. Kim, Y. H. Jang, D. E. Kim and T. Lee, Adv. Mater., 2015, 27, 2433-2439. 
6 C. Wang, X. Li, E. Gao, M. Jian, K. Xia, Q. Wang, Z. Xu, T. Ren and Y. Zhang, Adv. Mater., 2016, 28, 6640-6648.

7 Y. H. Kwon, S. W. Woo, H. R. Jung, H. K. Yu, K. Kim, B. H. Oh, S. Ahn, S.-Y. Lee, S.-W. Song, J. Cho and H. C. Shin, Adv. Mater., 2012, 24, 5192-5197.

8 W. Weng, Q. Sun, Y. Zhang, H. Lin, J. Ren, X. Lu, M. Wang and H. Peng, Nano Lett., 2014, 14, 3432-3438.

9 H. M. Kim, H. W. Kang, D. K. Hwang, H. S. Lim, B.-K. Ju and J. A. Lim, Adv. Funct. Mater., 2016, 26, 2706-2714.

10 D. Son, J. Lee, S. Qiao, R. Ghaffari, J. Kim, J. E. Lee, C. Song, S. J. Kim, D. J. Lee, S. W. Jun, S. Yang, M. Park, J. Shin, K. Do, M. Lee, K. Kang, C. S. Hwang, N. Lu, T. Hyeon and D.-H. Kim, Nat. Nanotechnol., 2014, 9, 397-404.

11 M. Stoppa and A. Chiolerio, Sensors, 2014, 14, 11957-11992.

12 E. R. Post, M. Orth, P. R. Russo and N. Gershenfeld, IBM Syst. J., 2000, 39, 840-860.

13 P. Rai, S. Oh, P. Shyamkumar, M. Ramasamy, R. E. Harbaugh and V. K. Varadan, J. Electrochem. Soc., 2014, 161, B3116B3150.

14 P. Gould, Mater. Today, 2003, 6, 38-43.

15 L. Cai, L. Song, P. Luan, Q. Zhang, N. Zhang, Q. Gao, D. Zhao, X. Zhang, M. Tu, F. Yang, W. Zhou, Q. Fan, J. Luo, W. Zhou, P. M. Ajayan and S. Xie, Sci. Rep., 2013, 3, 3048.

16 H. M. Lee, S. Y. Choi, A. Jung and S. H. Ko, Angew. Chem., Int. Ed., 2013, 52(30), 7718-7723.

17 J. Abel, J. Luntz and D. Brei, Smart Mater. Struct., 2012, 21, 085011.

18 Y. Cheng, R. Wang, J. Sun and L. Gao, ACS Nano, 2015, 9, 3887-3895.

19 Z. Wang, Y. Huang, J. Sun, Y. Huang, H. Hu, R. Jiang, W. Gai, G. Li and C. Z. Wang, ACS Appl. Mater. Interfaces, 2016, 8, 24837-24843.

20 X. Wu, Y. Han, X. Zhang and C. Lu, ACS Appl. Mater. Interfaces, 2016, 8, 9936-9945.

21 X. Liao, Q. Liao, Z. Zhang, X. Yan, Q. Liang, Q. Wang, M. Li and Y. Zhang, Adv. Funct. Mater., 2016, 26, 3074-3081.

22 Y. Wei, S. Chen, Y. Lin, X. Yuana and L. Liu, J. Mater. Chem. C, 2016, 4, 935-943.

23 Y. Tai and G. Lubineau, Adv. Funct. Mater., 2016, 26, 40784084.

24 J. Ge, L. Sun, F.-R. Zhang, Y. Zhang, L.-A. Shi, H.-Y. Zhao, H.-W. Zhu, H.-L. Jiang and S.-H. Yu, Adv. Mater., 2015, 28, 722-728.

25 S. Takamatsu, T. Kobayashi, N. Shibayama, K. Miyake and T. Itoh, Sens. Actuators, A, 2012, 184, 57-63.

26 S. Takamatsu, T. Yamashita, T. Imai and T. Itoh, Sens. Actuators, A, 2014, 220, 153-158.
27 M. Zhang, C. Wang, Q. Wang, M. Jian and Y. Zhan, ACS Appl. Mater. Interfaces, 2016, 8, 20894-20899.

28 H. Chang, G. Wang, A. Yang, X. Tao, X. Liu, Y. Shen and Z. Zheng, Adv. Funct. Mater., 2010, 20, 2893-2902.

29 F. Ochanda and W. E. Jones, Langmuir, 2005, 21, 1079110796.

30 J. Foroughi, G. M. Spinks, S. Aziz, A. Mirabedini, A. Jeiranikhameneh, G. G. Wallace, M. E. Kozlov and R. H. Baughman, ACS Nano, 2016, 10, 9129-9135.

31 J. T. Han, S. Choi, J. I. Jang, S. K. Seol, J. S. Woo, H. J. Jeong, S. Y. Jeong, K.-J. Baeg and G.-W. Lee, Sci. Rep., 2015, 5, 9300. 32 S. Seyedin, J. M. Razal, P. C. Innis, A. Jeiranikhameneh, S. Beirne and G. G. Wallace, Appl. Mater. Interfaces, 2015, 7, 21150-21158.

33 K. Koziol, J. Vilatela, A. Moisala, M. Motta, P. Cunniff, M. Sennett and A. Windle, Science, 2007, 318, 1892-1895.

34 X.-H. Zhong, Y.-L. Li, Y.-K. Liu, X.-H. Qiao, Y. Feng, J. Liang, J. Jin, L. Zhu, F. Hou and J.-Y. Li, Adv. Mater., 2010, 22, 692696.

35 X. Zhang, Q. Li, T. G. Holesinger, P. N. Arendt, J. Huang, P. D. Kirven, T. G. Clapp, R. F. DePaula, X. Liao, Y. Zhao, L. Zheng, D. E. Peterson and Y. Zhu, Adv. Mater., 2007, 19, 4198-4201.

36 S. Y. Kim, S. Park, H. W. Park, D. H. Park, Y. Jeong and D. H. Kim, Adv. Mater., 2015, 27, 4178-4185.

37 M. Zu, Q. Li, G. Wang, J.-H. Byun and T.-W. Chou, Adv. Funct. Mater., 2013, 23, 789-793.

38 J. A. Dobrzynska and M. A. M. Gijs, Sens. Actuators, A, 2012, 173, 127-135.

39 H. B. Muhammad, C. Recchiuto, C. M. Oddob, L. Beccai, C. J. Anthony, M. J. Adams, M. C. Carrozza and M. C. L. Ward, Microelectron. Eng., 2011, 88, 1811-1813.

40 S. C. B. Mannsfeld, B. C.-K. Tee, R. M. Stoltenberg, C. V. H.-H. Chen, S. Barman, B. V. O. Muir, A. N. Sokolov, C. Reese and Z. Bao, Nat. Mater., 2010, 9, 859-864.

41 S. Takamatsu, T. Kobayashi, N. Shibayama, K. Miyake and T. Itoh, Sens. Actuators, A, 2012, 184, 57-63.

42 L. M. Castano and A. B. Flatau, Smart Mater. Struct., 2014, 23, 053001.

43 B. Nie, R. Li, J. D. Brandt and T. Pan, Lab Chip, 2014, 14, 1107-1116.

44 M. L. Jin, S. Park, Y. Lee, J. H. Lee, J. Chung, J. S. Kim, J.-S. Kim, S. Y. Kim, E. Jee, D. W. Kim, J. W. Chung, S. G. Lee, D. Choi, H.-T. Jung and D. H. Kim, Adv. Mater., 2017, 29, 1605973. 